\title{
Characterization of infiltrating macrophages in high glucose-induced peritoneal fibrosis in rats
}

\author{
WENXUE HU*, ZONGPEI JIANG* , YU ZHANG, QINGHUA LIU, \\ JINJIN FAN, NING LUO, XIUQING DONG and XUEQING YU
}

\begin{abstract}
Department of Nephrology, The First Affiliated Hospital, Sun Yat-Sen University, Guangzhou 510080, P.R. China
\end{abstract}
Received January 12, 2012; Accepted April 10, 2012

DOI: $10.3892 / \mathrm{mmr} .2012 .890$

\begin{abstract}
Alternatively activated macrophages (M2 macrophages) are involved in tissue remodeling and fibrosis, but their effects on peritoneal fibrosis (PF) induced by high glucose peritoneal dialysate have yet to be fully established. In this study, PF was induced in male Sprague-Dawley rats by intraperitoneal injection with Lactate-G4.25\% dialysate (20 ml/rat/day) for 4 weeks. Control rats were given an intraperitoneal injection with saline. Establishment of the PF model was verified by Masson's trichrome and H\&E staining. M1 macrophages (co-localization of CD68 and CCr7) and M2 macrophages (co-localization of CD68 and CD206) was assayed by immunofluorescence and immunohistochemistry. The levels of dialysate cytokines driving macrophage differentiation (including IFN- $\gamma$, IL-2 and IL-4) were detected by ELISA. The expression of transforming growth factor (TGF)- $\beta$, p-Smad3, p-Smad2/3 and Smad7 in M2 macrophages (co-localization of CD68, CD206 and TGF- $\beta$, or p-Smad3, or p-Smad2/3, or Smad7) was measured by immunofluorescence. We found that PF rats had significantly thicker peritoneal membranes compared to control rats, indicating the successful establishment of our PF model. Compared to controls, PF rats had more peritoneal macrophages $\left(\mathrm{CD}^{\circ} 8^{+}\right.$cells), more peritoneal M1 macrophages and a greater percentage of peritoneal M2 macrophages. PF rats also had significantly greater levels of dialysate cytokine IL-4, which promotes differentiation to M2 macrophages, higher expression levels of TGF- $\beta$ in peritoneal M2 macrophages, upregulation of phosphorylated Smad3 and Smad2/3, and downregulation of Smad7 in peritoneal M2 macrophages. Our results indicate that M2 macrophages may play an important role in PF induced by high glucose, and that
\end{abstract}

Correspondence to: Professor Xueqing Yu, Key Laboratory of Nephrology, The First Affiliated Hospital, Sun Yat-Sen University, Ministry of Health, 58th Zhongshan Road II, Guangzhou 510080, P.R. China

E-mail: yuxq@mail.sysu.edu.cn

\section{*Contributed equally}

Key words: M1 macrophages, M2 macrophages, peritoneal fibrosis, transforming growth factor- $\beta$, Smad the cytokine environment in the abdominal cavities of PF rats promotes differentiation to M2 macrophages. The function of M2 macrophages in PF may be related to the TGF- $\beta / \mathrm{Smad}$ signaling pathways.

\section{Introduction}

Peritoneal dialysis (PD) is one of the renal replacement therapies for patients with end-stage renal diseases $(1,2)$. However, the peritoneal membranes of PD patients are continually exposed to non-biocompatibility PD solution, especially high glucose, and repeated peritonitis causes chronic inflammation, eventually leading to peritoneal fibrosis (PF) $(3,4)$. PF is a serious complication of long-term PD patients, resulting in ultrafiltration failure, increased solute transport and ultimately discontinuation of treatment $(5,6)$. Several studies concerning the mechanism of PF focus mainly on the epithelial-to-myofibroblast transition of peritoneal mesothelial cells and the functional alteration of fibroblasts $(7,8)$, but the role of macrophage differentiation in the pathogenesis of PF remains unknown.

Macrophages differentiate into functionally distinct immunological populations depending on the cytokine environment. The M1 phenotype (classically activated) macrophages are thought to be induced by interferon (IFN) $-\gamma$ and lipopolysaccharide (LPS), which features the production of inducible nitric oxide synthase (iNOS) and upregulates the expression of macrophage MHC class II and CC (CC motif) chemokine receptor 7 (CCr7) (9-11). Simultaneously, M1 produce the INF- $\gamma$ and tumor necrosis factor (TNF)- $\alpha$ cytokines. These pro-inflammatory cytokines and M1 macrophages function predominantly in inflammation, tissue damage, in the killing of intracellular microbes and increased tumoricidal microbes (12-14).

Nevertheless, the M2 phenotype (alternatively activated) macrophages are induced under the influence of Th2 cytokines, and, in particular, interleukin (IL)-4 and IL-13. This phenotype features upregulation of the expression of surface molecules, such as CD206 (also known as mannose receptor) and scavenger receptors (CD163) (10). More importantly, this specific macrophage subset is the main source of cytokines and chemokines, such as IL-10 and transforming growth factor (TGF)- $\beta(12,13)$. Through the cytokine pathways mentioned above, M2 may play an important role in the downregulation of inflammation, tissue remodeling, elimination of tissue debris and apoptotic bodies, as well as in the induction of 
fibrosis $(15,16)$. Moreover, several researchers reported that M2 macrophages promote fibrosis by producing TGF- $\beta$ and metalloproteinase (17). In turn, the TGF- $\beta /$ smad pathway may have a significant impact on fibrosis (18-20).

However, would the number and percentage of M1/ M2 macrophages be altered in PF models induced by high glucose dialysate? What about the cytokine environment driving macrophage differentiation? Is there any relationship between M2 macrophage differentiation and the TGF- $\beta / \mathrm{smad}$ signaling pathway in PF? These issues are not well known. We hypothesized that M2 may be increased in PF models induced by high glucose dialysate, cytokines driving M2 macrophage differentiation may be predominant and the TGF- $\beta /$ smad signaling pathway in M2 macrophages may be activated in PF models. In the present study, we tested this hypothesis in a rat model with intraperitoneal Lactate-G4.25\% dialysate.

\section{Materials and methods}

Materials. Lactate-G4.25\% dialysate was purchased from Baxter Healthcare, USA. Mouse anti-rat CD68, rabbit antirat CD206 and rabbit anti-rat p-Smad7 were purchased from Abcam, USA. Mouse anti-rat CCr7 was purchased from Cell Application, and rabbit anti-rat $\mathrm{p}-\mathrm{Smad} 2 / 3$ from Santa Cruz, USA. Rabbit anti-rat TGF- $\beta$ was purchased from Millipore, USA, and rabbit anti-rat p-Smad3 from CST, USA. HRP rabbit/mouse was purchased from Dako, Denmark. Alexa Fluor 488 goat anti-rabbit IgG, goat anti-rabbit 543 and goat anti-rabbit 633 were purchased from Invitrogen, USA. Normal bovine and rabbit blocking serum were purchased from Boster Biological Technology Company, China. 4',6-Diamindino-2phenylindole dihydrochloride (DAPI) was purchased from Sigma, UK. Aqueous mounting medium was purchased from R\&D, USA. INF- $\gamma$, IL-2 and IL-4 rat ELISA kits were purchased from Bender Medsystems, USA. QuantiChrom Arginase Assay kit was purchased from BioAssay, USA.

Animals. All rats were purchased from the Experimental Animal Centre of the Guangdong Province and raised in the Experimental Animal Centre at the Northern Campus of Sun Yat-Sen University. Experiments were conducted in accordance with animal care guidelines. A total of 12 male Sprague-Dawley rats weighing 120-150 g were randomly assigned to two groups: 6 rats received daily intraperitoneal (i.p.) injections of Lactate-G4.25\% dialysate $(100 \mathrm{ml} / \mathrm{kg})$ containing $4.25 \%$ glucose, and comprised the PF group, and another 6 rats received daily i.p. injections of the same volume of saline and were used as the normal control. Rats were sacrificed 4 weeks after the initiation of the i.p. injections. Avoiding the needle puncture sites, the parietal and visceral layers of the peritoneum were separately collected, fixed in paraformaldehyde at $4^{\circ} \mathrm{C}$ for $12 \mathrm{~h}$, washed overnight in $7 \%$ sucrose phosphate-buffered saline (PBS) and embedded in paraffin. The PD solution in the abdominal cavity of the rats was obtained and centrifuged at $4^{\circ} \mathrm{C}$ at $12,000 \mathrm{rpm}$ for $10 \mathrm{~min}$, and supernatants were stored at $-80^{\circ} \mathrm{C}$. The visceral peritoneum was frozen in liquid nitrogen and stored at $-80^{\circ} \mathrm{C}$.

Histology and immunocytochemistry. Both parietal and visceral peritoneal tissues were collected at 4 weeks after the initiation of
$\mathrm{PD}$ and fixed in paraformaldehyde for $12 \mathrm{~h}$. Paraffin-embedded sections $(5 \mu \mathrm{m})$ were cut and stained with H\&E and Masson's trichrome. For confocal imaging analysis, $8-\mu \mathrm{m}$ paraffinembedded sections were cut and dehydrated in xylene and a graded alcohol series, followed by microwave antigen retrieval. Sections were blocked with $1 \%$ albumin bovine fraction and normal goat serum for $30 \mathrm{~min}\left(37^{\circ} \mathrm{C}\right)$, and then incubated with primary antibody overnight at $4^{\circ} \mathrm{C}$, washed in PBS and incubated with Alexa Fluor-labeled secondary antibodies at $37^{\circ} \mathrm{C}$ for $30 \mathrm{~min}$. Two or three primary antibodies were co-dyed by the same protocol mentioned above. Next, sections were washed in PBS, incubated with DAPI for nuclear staining, sealed with aqueous mounting medium and viewed under a Zeiss LSM 510 confocal imaging system (Zeiss, Germany). Five fields were randomly selected and viewed; the number of CD68-, CD68- and CCr7- (M1 macrophage subtype) or CD68- and CD206-positive cells (M2 macrophages subtype) and intensity of fluorescence in each field of view were incorporated into the semiquantitative estimation of fluorescence intensity.

ELISA. The cytokine profiles (including INF- $\gamma$, IL-2 and IL-4) of the rat PD solution supernatant were determined by the rat ELISA kit, as described by the manufacturer. The estimated sensitivity of human INF- $\gamma$, IL-2 and IL-4 ELISAs were 9.9, 11 and $0.2 \mathrm{pg} / \mathrm{ml}$, respectively.

Statistical analysis. All obtained numerical data were shown as the means \pm SD. Comparisons between groups were performed using independent samples T test. Statistical t-test calculations were processed using the SPSS 16.0 software (SPSS Inc., USA). $\mathrm{P}<0.05$ denoted a statistically significant difference.

\section{Results}

Change of peritoneum in PF model rats. As shown in Fig. 1, significantly thicker peritoneum was found in the PF model induced by Lactate-G4.25\% dialysate compared to that in the normal control (232. \pm 34.3 vs. $54.3 \pm 11.5 \mu \mathrm{m}, \mathrm{P}<0.01)$. Reduced mesothelial cells and accumulation of collagen matrix in the submesothelial area were also found in the PF model rats.

\section{Macrophage accumulation in the peritoneum of PF model rats.} Immunohistochemical staining results showed that the number of macrophages $\left(\mathrm{CD} 68^{+}\right.$cells) in the peritoneal membranes of the PF model rats were significantly increased compared to those in the normal control (Fig. 2). Indirect immunofluorescence staining with confocal photomicrographs showed that the number of M1 infiltration in the PF model rats was significantly enhanced compared to that in the normal control rats. However, there was no difference in the percentage of M1 in total macrophages between the control and the PF model rats (Fig. 3). By contrast, co-localization signals of CD68 and CD206 were identified as M2 macrophages. Both the absolute number and percentage of M2 in total macrophages were significantly increased in the PF model rats compared to that in the normal control rats (Fig. 4).

Expression of cytokines related to macrophage differentiation or transverse in PD effluent of the PF model and control rats. IFN- $\gamma$ and IL-2, both driving M1 macrophage differentiation, 

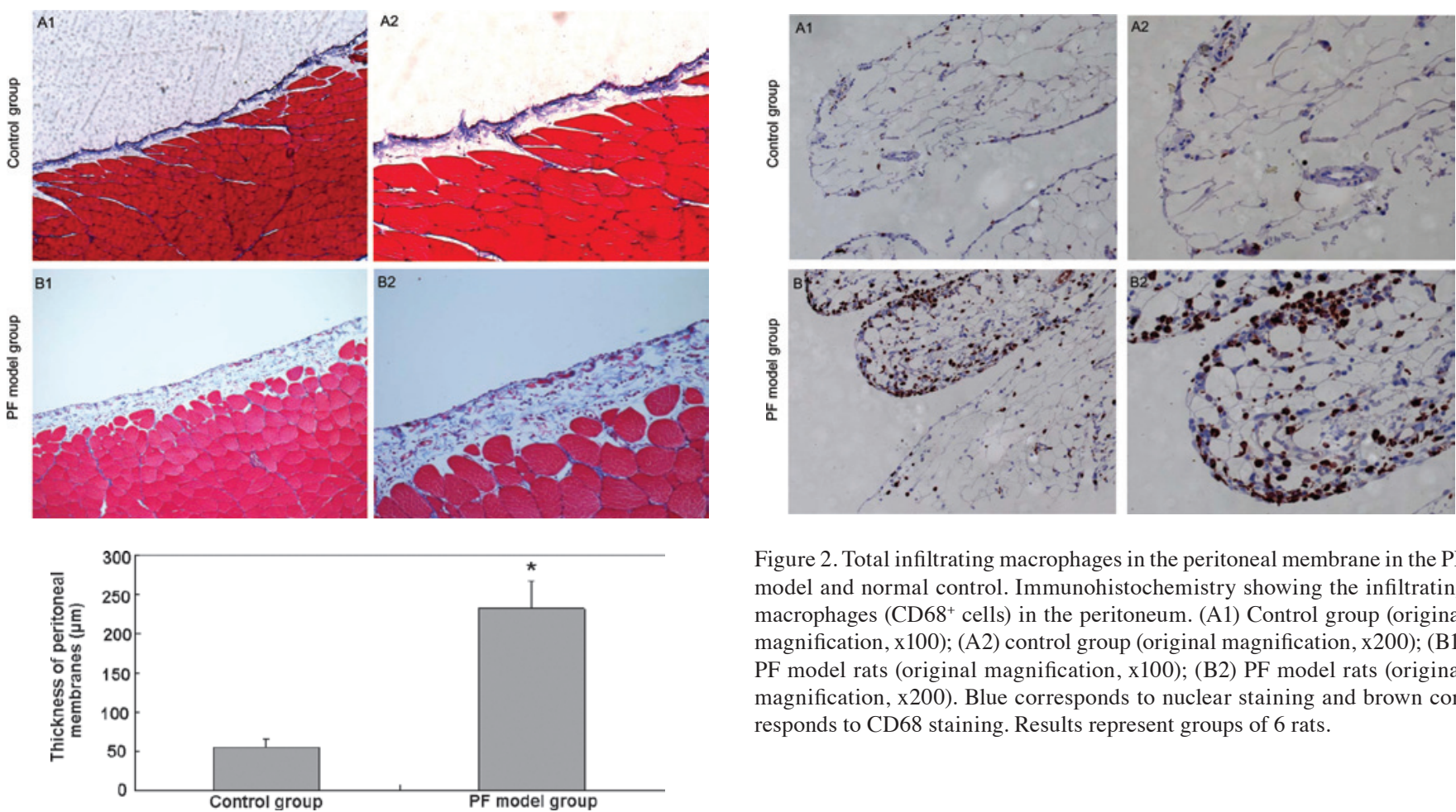

Figure 2. Total infiltrating macrophages in the peritoneal membrane in the PF model and normal control. Immunohistochemistry showing the infiltrating macrophages $\left(\mathrm{CD} 68^{+}\right.$cells) in the peritoneum. (A1) Control group (original magnification, x100); (A2) control group (original magnification, x200); (B1) PF model rats (original magnification, x100); (B2) PF model rats (original magnification, $\mathrm{x} 200$ ). Blue corresponds to nuclear staining and brown corresponds to CD68 staining. Results represent groups of 6 rats.

Figure 1. Thickness of the peritoneal membrane in PF model rats and normal control. Masson's trichrome staining of day-28 parietal peritoneal tissues. (A1) Control group (original magnification, x100); (A2) control group (original magnification, x200); (B1) PF model rat (original magnification, x100); (B2) PF model rat (original magnification, x200). Results represent groups of 6 rats.

were significantly increased in the PF model rats compared to the normal control. Moreover, IL-4 driving M2 macrophage

differentiation was also significantly increased in the PF model rats compared to the normal control. However, the ratio of IFN- $\gamma /$ IL-4 was significantly decreased in the PF model rats compared to that in the normal control (Fig. 5).

Activation of TGF- $\beta /$ Smad signaling in M2 macrophages. Double immunostaining with confocal photomicrographs showed that TGF- $\beta 1$ expression in M2 macrophages was markedly increased in the PF model rats compared to the normal
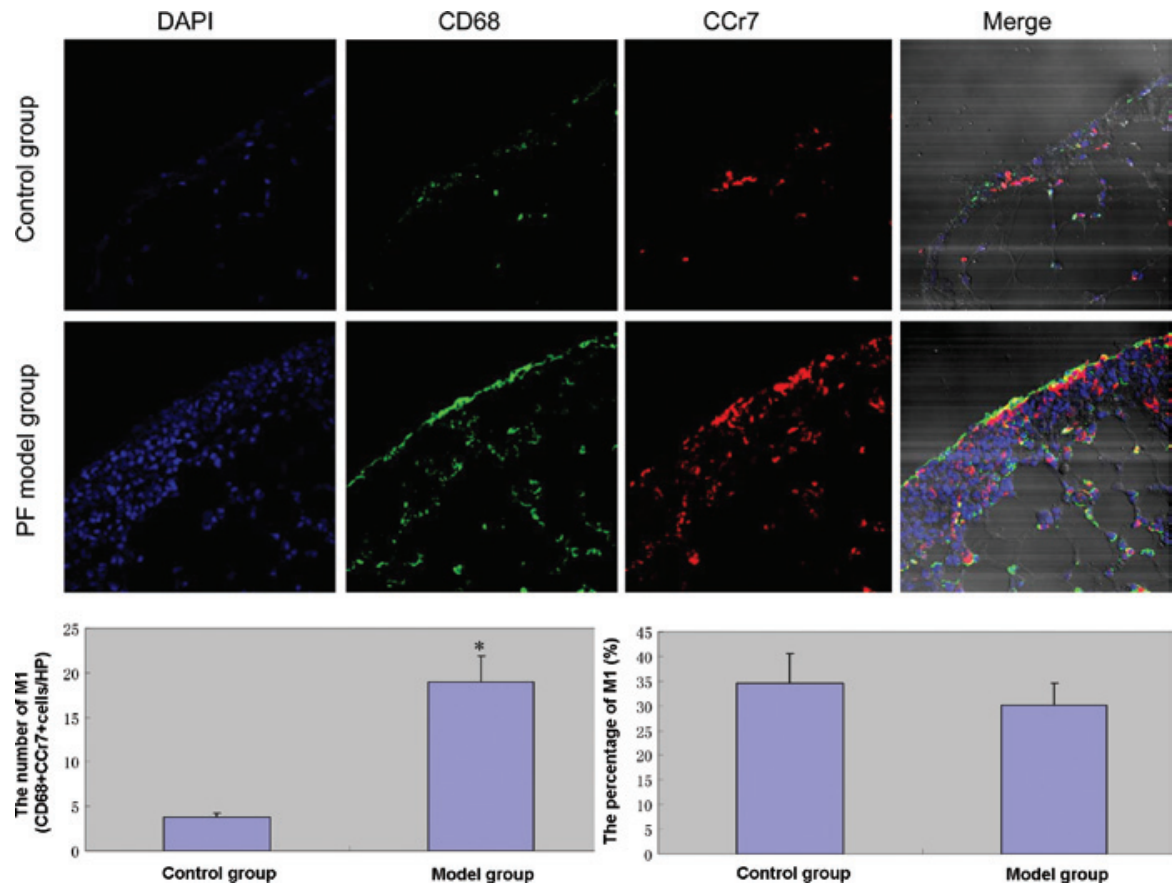

Figure 3. Number and percentage of M1 macrophages in the peritoneum of the control and PF model groups. Confocal photomicrographs show that the number of M1 macrophages (co-localization signals of CD68 and CCr7) was significantly increased in the PF model rats. The difference in the percentage of M1 was not significant between the control and the PF model groups. Blue corresponds to nuclear staining, red corresponds to CD68 staining and green corresponds to $\mathrm{CCr} 7$ staining. Results are the means \pm SEM for a group of 6 rats. $\mathrm{P}<0.01$ vs. normal control. HP, high-power field. 

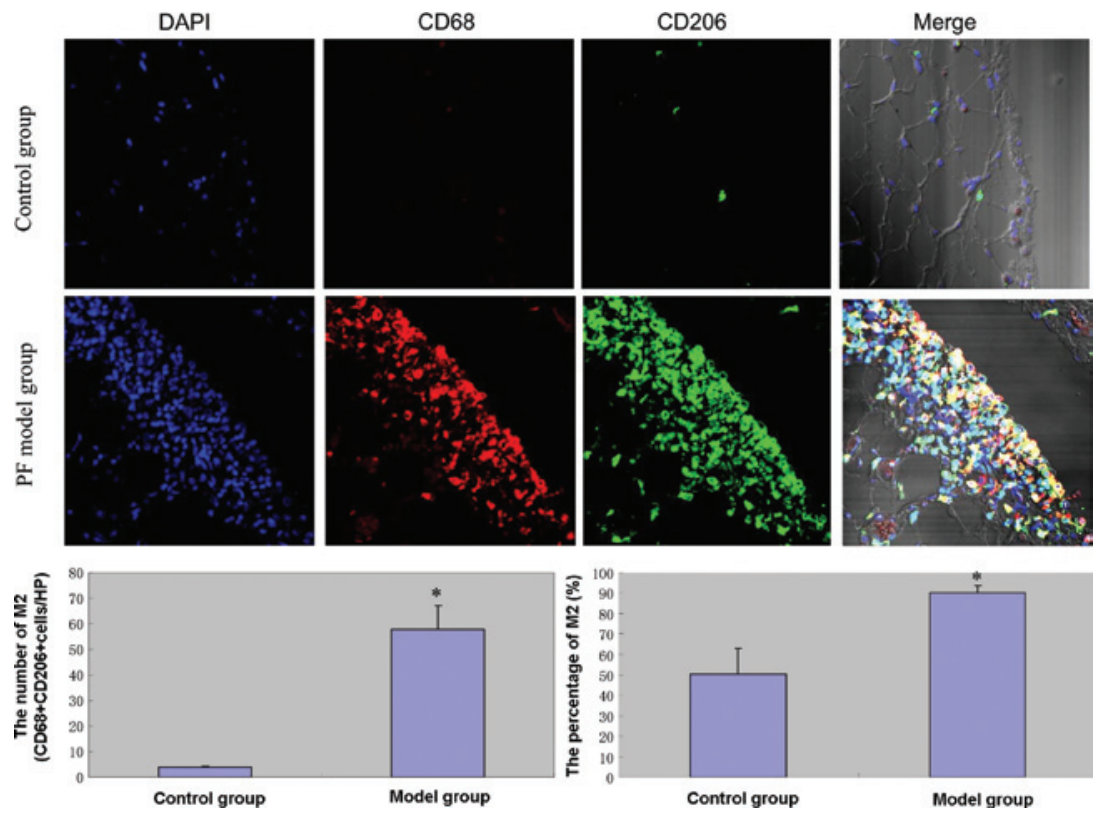

Figure 4. Number and percentage of M2 macrophages in the peritoneum of the control and PF model groups. Confocal photomicrographs show the number and percentage of M2 macrophages (co-localization signals of CD68 and CD206) are significantly increased in PF model rats. Blue corresponds to nuclear staining, red corresponds to CD68 staining and green corresponds to CD206 staining. Results are the means \pm SEM for a group of 6 rats. " $\mathrm{P}<0.01$ vs. normal control. HP, high-power field.
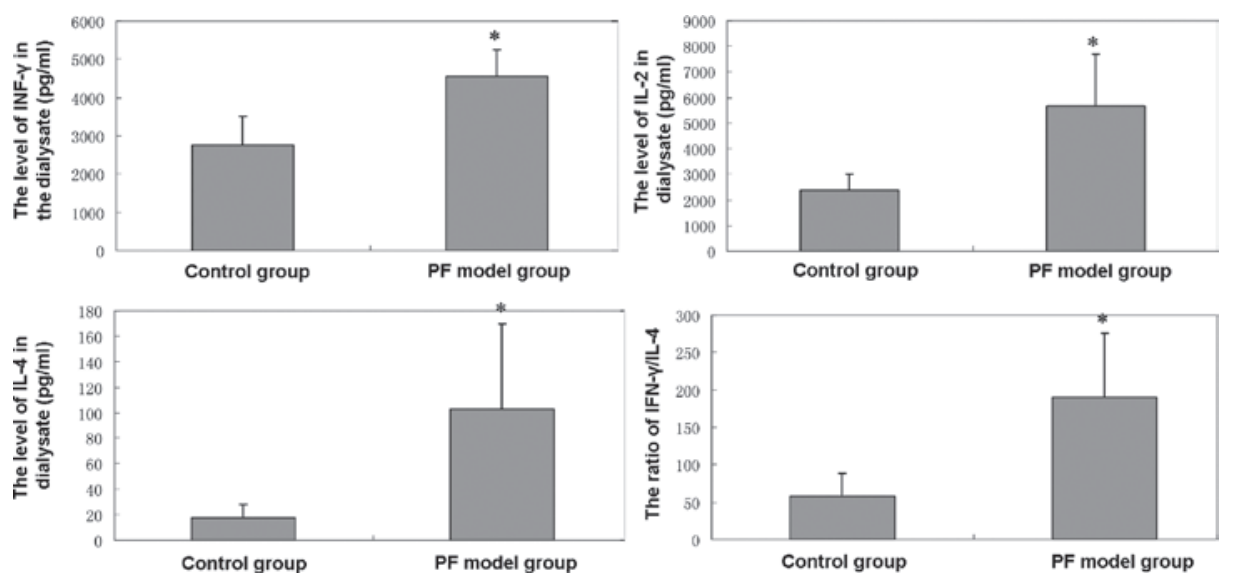

Figure 5. Cytokines driving macrophages in the dialysate of rats in the control and PF model groups. The level of driving M1 macrophage cytokines INF- $\gamma$ and IL-2, and the level of driving M2 macrophage cytokine IL-4 were detected by ELISA. The ratio of IFN- $\gamma /$ IL-4 was significantly increased in the PD model group. Each bar represents the mean \pm SEM for a group of 6 rats. " $\mathrm{P}<0.01$ vs. normal control.
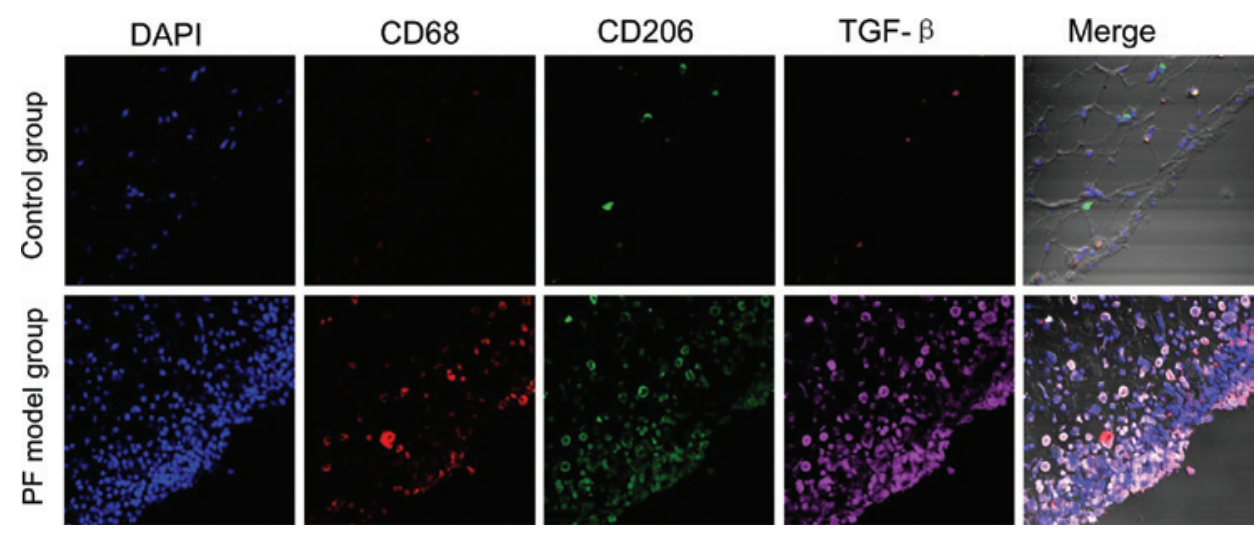

Figure 6. Expression of TGF- $\beta$ in peritoneal M2 macrophages in the PF model and normal control. Confocal photomicrographs show the TGF- $\beta$ protein in peritoneal M2 macrophages (co-localization signals of CD68, CD206 and TGF- $\beta$ ) of control and PF model rats. Blue corresponds to nuclear staining, red corresponds to CD68 staining, green corresponds to CD206 staining and purple corresponds to TGF- $\beta$. 

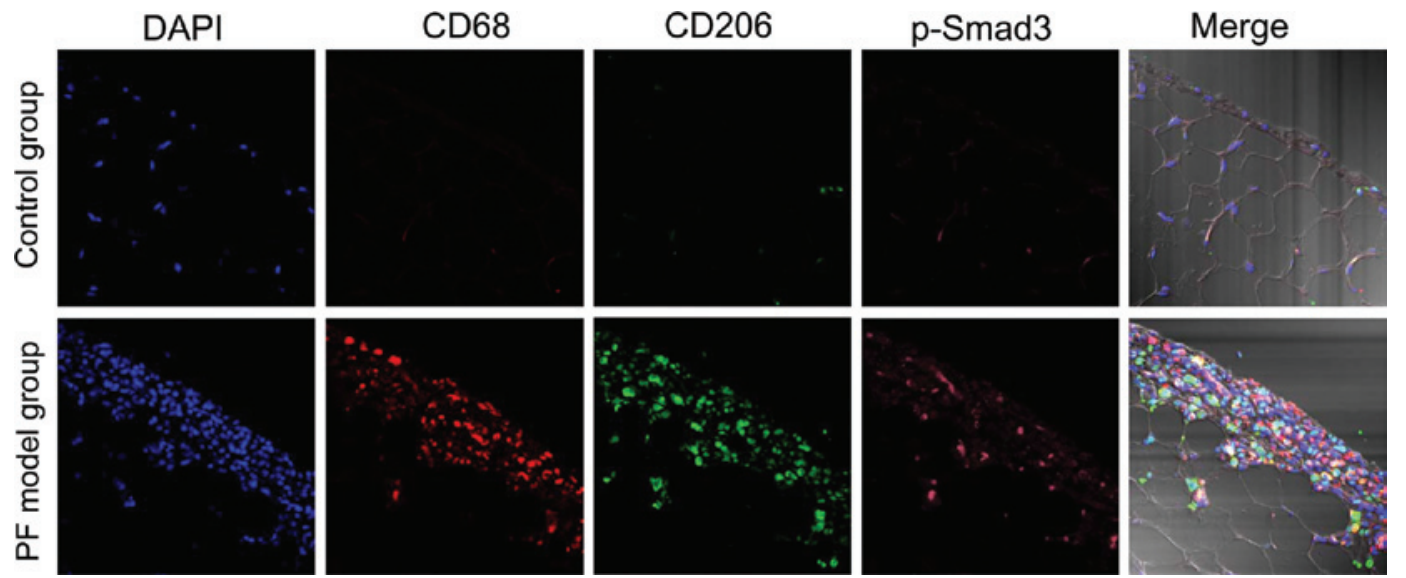

Figure 7. Phosphorylation of Smad3 in peritoneal M2 macrophages in the PF model and normal control. Confocal photomicrographs show the Smad3 protein in peritoneal M2 macrophages (co-localization signals of CD68, CD206 and Smad3) of control and PF model rats. Blue corresponds to nuclear staining, red corresponds to CD68 staining, green corresponds to CD206 staining and purple corresponds to Smad3.
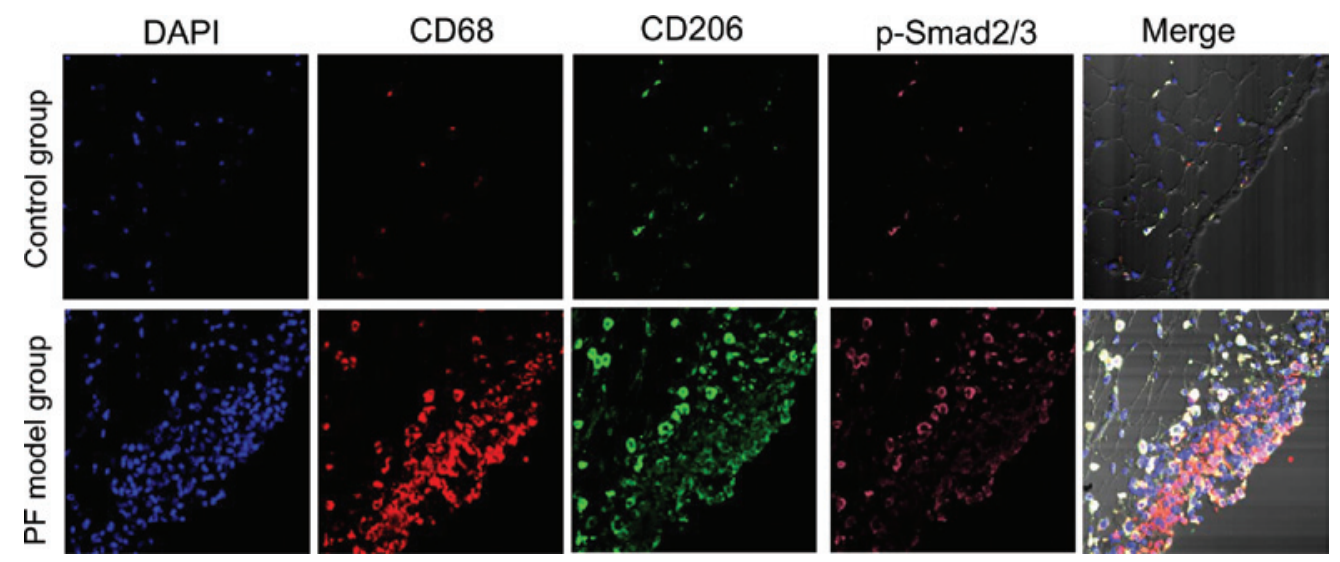

Figure 8. Phosphorylation of Smad2/3 in peritoneal M2 macrophages in the PF model and normal control. Confocal photomicrographs show the Smad2/3 protein in peritoneal M2 macrophages (co-localization signals of CD68, CD206 and Smad2/3) of control and PF model rats. Blue corresponds to nuclear staining, red corresponds to CD68 staining, green corresponds to CD206 staining and purple corresponds to Smad2/3.

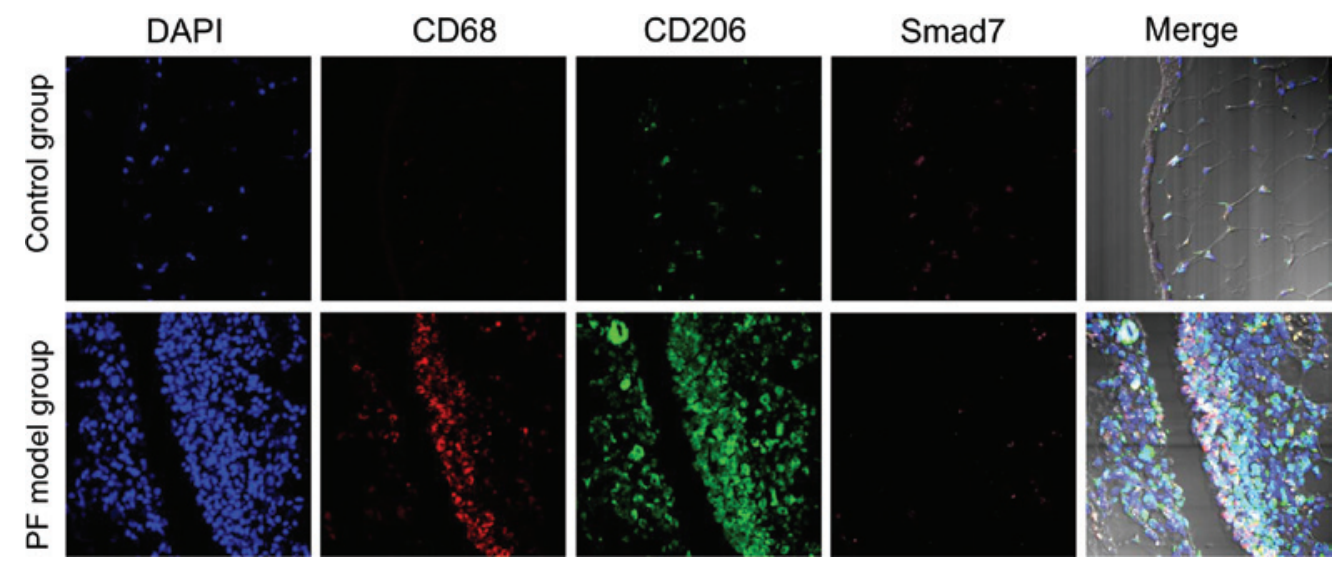

Figure 9. Expression of Smad7 in peritoneal M2 macrophages. Confocal photomicrographs show the Smad7 protein in peritoneal M2 macrophages (colocalization signals of CD68, CD206 and Smad7) of control and PF model rats. Blue corresponds to nuclear staining, red corresponds to CD68 staining, green corresponds to CD206 staining and purple corresponds to Smad7.

rats (Fig. 6). Moreover, immunofluorescent staining showed that there was a marked increase in phosphorylated Smad3 and phosphorylated Smad2/3 cells translocated into nuclei of M2 macrophages after treatment with Lactate-G4.25\% dialysate (Figs. 7 and 8). Furthermore, expression of Smad7 in M2 macrophages was significantly decreased in the PF model rats (Fig. 9). 


\section{Discussion}

In the present study, our data showed that the number of M1 macrophages and the number and percentage of M2 macrophages were significantly increased in the PF model rats induced by Lactate-G4.25\% dialysate. Cytokines contributing to M2 differentiation in the PF model rats were predominant. We also found that TGF- $\beta /$ Smad signals were activated in the peritoneal M2 macrophages of the PF model rats.

During PD, peritoneal cells are repeatedly exposed to a non-physiological hypertonic environment with high glucose content and low $\mathrm{pH}$. Chronic changes in the peritoneum with fibrosis develop after years of PD (21). Peritoneal inflammation is a key and initial step in the development of PF associated with long-term PD $(22,23)$. Macrophages are one of the main cell populations mediating inflammation, and play an important role in innate and adaptive immune responses $(24,25)$. Osada et al proved that CD68-positive cells (macrophages) in the peritoneum were marked in long-term PD patient samples (26). Our study showed that macrophages were significantly increased in the peritoneum of rats administered daily i.p. injections of Lactate-G4.25\% dialysate. These data indicate that macrophages may play a key role in the pathogenesis of fibrosis of peritoneum exposed to PD solution.

Macrophages are classically activated towards the M1 phenotype by LPS or IFN- $\gamma$, that promote active inflammation. de Souza et al demonstrated that high glucose increased macrophage activation by lipoteichoic acid, evidenced by exacerbated nitric oxide and TNF- $\alpha$ production (27). Similarly, macrophages in the peritoneum may be stimulated by dialysate containing high glucose and produce cytokines, such as TNF- $\alpha$. Subsequently, these cytokines exert an autocrine effect driving macrophages to M1. In addition, the interaction of high glucose and the protein of cells contribute to the production of glycation end-products (GEPs), which significantly impact the differentiation of macrophages. Ohashi et al investigated the effect of four distinct GEP subtypes on the expression of intracellular adhesion molecules and CD40 on monocytes, and the production of IFN- $\gamma$ and TNF- $\alpha$. These two are marker cytokines of M1 macrophages and thus GEP induced by the interaction of high glucose and cells may be a potential mechanism of peritoneal macrophages differentiating to M1 (28). We found that the number of M1 macrophages was significantly increased in the PF model rats, and these M1 macrophages may be induced by ways mentioned previously. However, there was no difference in the percentage of M1 between the control and the PF model rats, which indicates that M1 macrophages are not predominant in fibrosis of the peritoneum.

By contrast, macrophages are alternatively activated towards the M2 phenotype by stimulation with IL- 4 and IL-13. Several studies suggest that M2 macrophages play an important role in fibrosis of the lung, liver, skin and muscle fibrosis (16,29-32). Murphy et al analyzed bronchoalveolar lavage or spontaneously expectorated sputum samples from 48 cystic fibrosis patients. Their data showed that surface expression of M2 macrophages was upregulated (29). Heymann et al conducted a study on the relationship between Kupffer cells and liver fibrosis. They demonstrated that M2 macrophages were not only critical to the progress of liver fibrosis, but were also important for reversing the damage (30). Desguerre et al performed a study on Duchenne muscular dystrophy and found that myofibers exhibited oxidative stress-induced protein alterations and became separated from capillaries by fibrosis, which was associated with the increase of M2 macrophages (16). Thus, they believed that M2 macrophages inhibit myogenesis, while promoting cellular collagen production, playing a key role in myofibrosis. Higashi-Kuwata et al found that in the skin from systemic sclerosis patients, the number of $\mathrm{CD}_{163^{+}}$ or $\mathrm{CD} 204^{+}$cells between the collagen fibers was significantly larger than that in healthy controls (32). Our study showed that the number and percentage of peritoneal M2 macrophages were significantly increased in the PF model rats, which is in accordance with the studies mentioned previously. These data indicate that M2 macrophages may play crucial roles in the fibrosis of the peritoneum of rats receiving daily i.p. injections of Lactate-G4.25\% dialysate.

Macrophages are alternatively activated to M2 macrophages, which produce functional cytokines, including TGF- $\beta$ and IL-10 $(12,13)$. Several studies suggest that TGF- $\beta$ is a multifunctional cytokine central in the process of fibrogenesis and has been implicated in the initiation, progression and maintenance of PF $(33,34)$. Moreover, TGF- $\beta$ exhibits its bioactivities via serine/threonine kinase transmembrane receptors that phosphorylate its downstream mediators of the Smad family, including Smad2 and Smad3. Once activated, TGF- $\beta$ is also capable of inducing expression of an inhibitory Smad7, which in turn prevents Smad2/3 phosphorylation via its negative-feedback mechanism $(35,36)$. A previous study in our laboratory showed that prevention of PF by overexpression of Smad7 was associated with the inhibition of phosphorylation of Smad2/3, downstream of the TGF- $\beta$ signaling pathway, as well as TGF- $\beta$ expression (20). In the present study, the expression of TGF- $\beta$ in peritoneal M2 was significantly increased in PF rats. Moreover, phosphorylation of Smad3 and Smad2/3 was markedly upregulated, while Smad7 was downregulated in peritoneal M2 of those PF rats. All these results demonstrate that M2 macrophages having an effect on PF may be associated with the TGF- $\beta /$ Smad signaling pathways.

Taken together, we demonstrated that M2 macrophages play an important role in peritoneal fibrosis induced by dialysate containing high glucose. Moreover, in fibrotic peritoneum, the cytokine environment is advantageous for M2 differentiation. Notably, M2 macrophage function in PF may be associated with the TGF- $\beta /$ Smad signaling pathways. These data also indicate that blockade of M2 macrophage differentiation may be a mechanism by which to prevent peritoneal fibrosis. The latter requires further study.

\section{Acknowledgements}

This study was supported by the Baxter's Renal Discoveries Extramural Grant Program (EGP GRANT no. 09AP012-OG), and the National Natural Science Foundation of China (grant no. 30771012).

\section{References}

1. Krediet RT: 30 years of peritoneal dialysis development: the past and the future. Perit Dial Int Suppl 2: 35-41, 2007. 
2. Oreopoulos DG and Yang X: Long-term survival on peritoneal dialysis is not a dream anymore: lessons from the East. Perit Dial Int 27: 410-412, 2007.

3. Di Paolo N and Sacchi G: The peritoneum during peritoneal dialysis. Perit Dial Int 20: 37-63, 2000.

4. Yung S and Chan TM: Preventing peritoneal fibrosis - insights from the laboratory. Perit Dial Int 23 (Suppl 2): 37-41, 2003.

5. Garosi G, Cappelletti F and Di Paolo N: Fibrosis and sclerosis: different disorders or different stages? Contrib Nephrol 150 62-69, 2006.

6. Sandoval P, Loureiro J, González-Mateo G, et al: PPAR- $\gamma$ agonist rosiglitazone protects peritoneal membrane from dialysis fluidinduced damage. Lab Invest 90: 1517-1532, 2010.

7. Oh EJ, Ryu HM, Choi SY, et al: Impact of low glucose degradation product bicarbonate/lactate-buffered dialysis solution on the epithelial-mesenchymal transition of peritoneum. Am J Nephrol 31: 58-67, 2010.

8. Kim YL: Update on mechanisms of ultrafiltration failure. Perit Dial Int Suppl 2: 123-127, 2009.

9. Gordon S: Alternative activation of macrophages. Nat Rev Immunol 3: 23-35, 2003.

10. Martinez FO, Sica A, Mantovani A and Locati M: Macrophage activation and polarization. Front Biosci 13: 453-461, 2008.

11. Badylak SF, Valentin JE, Ravindra AK, McCabe GP and Stewart-Akers AM: Macrophage phenotype as a determinant of biologic scaffold remodeling. Tissue Eng Part A 14: 1835-1842, 2008.

12. Mantovani A, Sica A and Locati M: Macrophage polarization comes of age. Immunity 23: 344-346, 2005.

13. Troidl C, Möllmann H, Nef H, et al: Classically and alternatively activated macrophages contribute to tissue remodelling after myocardial infarction. J Cell Mol Med 13: 3485-3496, 2009.

14. Ohri CM, Shikotra A, Green RH, Waller DA and Bradding P. Macrophages within NSCLC tumour islets are predominantly of a cytotoxic M1 phenotype associated with extended survival. Eur Respir J 33: 118-126, 2009.

15. Holt MP, Cheng L and Ju C: Identification and characterization of infiltrating macrophages in acetaminophen-induced liver injury. J Leukoc Biol 84: 1410-1421, 2008.

16. Desguerre I, Mayer M, Leturcq F, Barbet JP, Gherardi RK and Christov C: Endomysial fibrosis in Duchenne muscular dystrophy: a marker of poor outcome associated with macrophage alternative activation. J Neuropathol Exp Neurol 68: 762-773, 2009.

17. Vidal B, Serrano AL, Tjwa M, et al: Fibrinogen drives dystrophic muscle fibrosis via a TGFbeta/alternative macrophage activation pathway. Genes Dev 22: 1747-1752, 2008.

18. Nie J, Hao W, Dou X, et al: Effects of Smad7 overexpression on peritoneal inflammation in a rat peritoneal dialysis model. Perit Dial Int 27: 580-588, 2007.

19. Ng YY, Hou CC, Wang W, Huang XR and Lan HY: Blockade of $\mathrm{NF} \kappa \mathrm{B}$ activation and renal inflammation by ultrasound-mediated gene transfer of Smad7 in rat remnant kidney. Kidney Int 94: S83-S91, 2005
20. Wang W, Huang XR, Li AG, et al: Signaling mechanism of TGF- $\beta$ in prevention of renal inflammation: role of Smad7. J Am Soc Nephrol 16: 1371-1383, 2005.

21. Lai KN and Leung JC: Inflammation in peritoneal dialysis. Nephron Clin Pract 116: 11-18, 2010.

22. Kon M, Hirayama S, Horiuchi Y, et al: Profiles of inflammatory markers and lipoprotein subclasses in patients undergoing continuous ambulatory peritoneal dialysis. Clin Chim Acta 411: $1723-1727,2010$

23. Devuyst O, Margetts PJ and Topley N: The pathophysiology of the peritoneal membrane. J Am Soc Nephrol 21: 1077-1085, 2010.

24. Palomar R, López-Hoyos M, Morales P, et al: Analysis of peritoneal leukocyte population with different dialysis fluids. Clin Nephrol 72: 137-142, 2009.

25. Mehta A, Brewington R, Chatterji M, et al: Infection-induced modulation of $\mathrm{m} 1$ and $\mathrm{m} 2$ phenotypes in circulating monocytes: role in immune monitoring and early prognosis of sepsis. Shock 22: 423-430, 2004

26. Osada S, Hamada C, Shimaoka T, Kaneko K, Horikoshi S and Tomino Y: Alterations in proteoglycan components and histopathology of the peritoneum in uraemic and peritoneal dialysis (PD) patients. Nephrol Dial Transplant 24: 3504-3512, 2009.

27. de Souza LF, Jardim FR, Sauter IP, de Souza MM and Bernard EA: High glucose increases RAW 264.7 macrophages activation by lipoteichoic acid from Staphylococcus aureus. Clin Chim Acta 398: 130-133, 2008.

28. Ohashi K, Takahashi HK, Mori S, et al: Advanced glycation end products enhance monocyte activation during human mixed lymphocyte reaction. Clin Immunol 134: 345-353, 2010.

29. Murphy BS, Bush HM, Sundareshan V, et al: Characterization of macrophage activation states in patients with cystic fibrosis. J Cyst Fibros 9: 314-322, 2010.

30. Heymann F, Trautwein C and Tacke F: Monocytes and macrophages as cellular targets in liver fibrosis. Inflamm Allergy Drug Targets 8: 307-318, 2009.

31. Mathai SK, Gulati M, Peng X, et al: Circulating monocytes from systemic sclerosis patients with interstitial lung disease show an enhanced profibrotic phenotype. Lab Invest 90: 812-823, 2010.

32. Higashi-Kuwata N, Jinnin M, Makino T, et al: Characterization of monocyte/macrophage subsets in the skin and peripheral blood derived from patients with systemic sclerosis. Arthritis Res Ther 12: 128, 2010.

33. Takagawa S, Lakos G, Mori Y, Yamamoto T, Nishioka K and Varga J: Sustained activation of fibroblast transforming growth factor-beta/Smad signaling in a murine model of scleroderma. J Invest Dermatol 121: 41-50, 2003.

34. Tamaki K and Okuda S: Role of TGF-beta in the progression of renal fibrosis. Contrib Nephrol 139: 44-65, 2003.

35. Piek E, Heldin CH and Ten DP: Specificity, diversity, and regulation in TGF-beta superfamily signaling. FASEB J 13 2105-2124, 1999

36. Massagué J and Wotton D: Transcriptional control by the TGF- $\beta /$ Smad signaling system. EMBO J 19: 1745-1754, 2000. 